\title{
Pressurized intraperitoneal aerosol chemotherapy procedure for nonresectable peritoneal carcinomatosis: First Indian study
}

\author{
S. P. Somashekhar, K. R. Ashwin, C. Rohit Kumar, Amit Rauthan', Sushmita H. Rakshit ${ }^{2}$
}

\begin{abstract}
Background: Peritoneal carcinomatosis (PC) is a common evolution of abdominal cancers and is associated with poor prognosis.A few selected patients have option of cytoreductive surgery and hyperthermic intraperitoneal chemotherapy, but majority who are not eligible for curative approach can undergo pressurized intraperitoneal aerosol chemotherapy (PIPAC). It is an emerging field of research with major therapeutic potential. It is a safe and innovative approach, which enhances the effect of chemotherapy without major toxicity. Methods: Between June 2017 and December 20I7, 2 I PIPAC applications in seven patients with standard chemotherapy regimen every 6 weeks at $37^{\circ} \mathrm{C}$ and $12 \mathrm{mmHg}$ for 30 min was performed. The patients' demographics, perioperative findings, adverse events, and outcomes were prospectively recorded. Results: Twenty-one PIPAC administrations were performed in 7 patients with PC from various pathologies. The median hospital stay was I day. All the patients had symptomatic relief with complete resolution of ascites. There was no major perioperative complications. CTCAE Grades I and 2 were observed in three patients, for abdominal pain and nausea. Renal and hepatic functions were not impaired. Of the seven patients, one patient had complete histological remission; three patients had partial response, one had stable disease and one patient had no response with clinical progression. Conclusion: Our results show the feasibility and safety of PIPAC in Indian patients. The procedure has low morbidity with no mortality with the short learning curve. It can be easily adapted for Indian patients with diffuse PC as a palliative option apart from systemic chemotherapy.
\end{abstract}

Key words: Chemotherapy, cytoreductive surgery, hyperthermic intraperitoneal chemotherapy, Indian patients, intraperitoneal, peritoneal carcinomatosis, pressurized intraperitoneal aerosol chemotherapy

\section{Introduction}

Peritoneal carcinomatosis (PC) was regarded as a terminal disease with traditional palliative treatment options of systemic chemotherapy or palliative surgery having a poor outcome. ${ }^{[1,2]}$

Pressurized intraperitoneal aerosol chemotherapy (PIPAC) is a novel technique delivering normothermic chemotherapy into the abdominal cavity as an aerosol under pressure which has a documented positive outcome by counterbalancing the elevated tumoral interstitial fluid pressure ${ }^{[3-5]}$ and enhancing drug depth penetration with superior distribution. PIPAC is currently used for palliative setting in selected patients with trials ongoing.

\section{Methods}

PIPAC program for patients diagnosed with advanced PC was introduced at Manipal Comprehensive Cancer Center from June 2017. Training was provided to educate the healthcare providers about the technical and safety aspects of the procedure. All patients with histologically verified peritoneal metastasis secondary to mesothelioma, gastric/colorectal/ovarian cancer were presented in the interdisciplinary tumor board, and the indication for therapy was decided on a case-by-case basis. PIPAC was offered where option of cytoreductive surgery and hyperthermic intraperitoneal chemotherapy was not possible because of poor general condition (Eastern Cooperative Oncology Group [ECOG] >2), advanced peripheral component interconnect (PCI), and/or unresectablity because of diffuse small bowel involvement. Patients were eligible if they had blood and electrolyte counts, liver, renal, and cardiopulmonary function parameters within $10 \%$ of the normal range. All patients were counseled and their informed consent obtained. The Institutional review board and Ethics Committee approval was obtained. We report the technical aspects, our observations, and outcomes with PIPAC procedure in Indian patients.

\begin{tabular}{|l|}
\hline Access this article online \\
\hline Quick Response Code: \\
\\
Website: www.sajc.org \\
\hline DOI: 10.4103 /sajc.sajc_92_18 \\
\hline
\end{tabular}

Departments of Surgical Oncology and 'Medical Oncology, Manipal Comprehensive Cancer Center, Manipal Hospital, ${ }^{2}$ Department of Surgical Oncology, Manipal Hospital, Bengaluru, Karnataka, India Correspondence to: Dr. S. P. Somashekhar, E-mail: somusp@yahoo.com
Technique of pressurized intraperitoneal aerosol chemotherapy

All operations were performed under general anesthesia; a standard operating protocol was followed with emphasis on handling and exposure to chemotherapy. A antibiotic prophylaxis with a single dose of cefuroxime $1.5 \mathrm{~g}$ intravenous (IV) was administered $30 \mathrm{~min}$ before surgery. After insufflation of a $12 \mathrm{mmHg}$ pneumoperitoneum (with open access or Veres needle), two $5 \mathrm{~mm}$ trocars were inserted into the abdominal wall. Ascites was aspirated and sent for cytology testing. Extent of PC was determined based on PCI score. A centimetric local peritonectomy was performed for peritoneal biopsies in all cases to improve accuracy of anatomopathology. The intraperitoneal chemotherapy was given as per standard doses. ${ }^{[6-8]}$ The generation of aerosol requires a disposable 9 -mm microinjector (Capnopen ${ }^{\circledR}$, Capnomed, Villingendorf, Germany) which was connected to an IV high-pressure injector (Angiomat Illumena Injector ${ }^{\circledR}$, Liebel Flarsheim, USA) and inserted into the abdomen through a $12 \mathrm{~mm}$ access port. Safety measures were taken to prevent any exposure of drugs to the operating team. The procedure was performed in an operating room equipped with laminar airflow. Tightness of the abdomen was documented through a zero flow of $\mathrm{CO}_{2}$ to prevent operation theater (OT) contamination. The chemotherapy injection was remote-controlled and nobody remained in the operating room during the application. The laparoscopic and anesthesia monitors are oriented toward the OT door window to facilitate monitoring by the doctors from outside [Figure 1].

Aerosol flow rate was $30 \mathrm{ml} / \mathrm{min}$, and maximal upstream pressure was 200 psi as per recommendation. The therapeutic

This is an open access journal, and articles are distributed under the terms of the Creative Commons Attribution-NonCommercial-ShareAlike 4.0 License, which allows others to remix, tweak, and build upon the work non-commercially, as long as appropriate credit is given and the new creations are licensed under the identical terms.

For reprints contact: reprints@ medknow.com

How to cite this article: Somashekhar SP, Ashwin KR, Kumar RC, Rauthan A, Rakshit SH. Pressurized intraperitoneal aerosol chemotherapy procedure for nonresectable peritoneal carcinomatosis: First Indian study. South Asian J Cancer 2019;8:27-30. 
capnoperitoneum was then maintained for $30 \mathrm{~min}$. Then, the chemotherapy aerosol was released safely through a closed aerosol waste system into the air waste system of the hospital or by a Buffalo filter. Trocars were retracted, and laparoscopy was ended. No drainage of the abdomen was placed. Patients were discharged the following day in the absence of adverse effects [Figure 2]. ${ }^{[9]}$

\section{Results}

A total of 21 successful PIPAC procedures were carried out in seven patients with PC. The primary tumor site was one colorectal cancer $(14.2 \%)$, two epithelial ovarian cancer $(28.7 \%)$, two mesotheliomas $(28.7 \%)$, one primary peritoneal cancer (PPC) $(14.2 \%)$ and one gastric cancer (14.2\%); There were 5 males and three females. Two patients had ECOG performance of 2, and the rest had performance status of $0-1$. Three patients were symptomatic with abdominal pain and/or subacute obstruction. All patients were pretreated with mean number of 1.3 lines per patient (range 1-3).

Patient's characteristics and preoperative details are summarized in Table 1. In two patients, entry to the abdominal cavity was difficulty due to adhesions and had to undergo minimal adhesiolysis for port access. Mean operating time was $98.6 \mathrm{~min}(80-120)$. No intraoperative complications or allergic reactions were noted. The mean hospital stay was 1.85 (range 1-3), and median stay was 1 day. Mean PCI was 17.1 (range 11-23). PIPAC was well tolerated with acute and cumulative local toxicities of PIPAC under control and no severe side effects observed. Adverse events were noted and graded as per CTCAE. Abdominal pain CTCAE $\leq 2$ was noted in $3 / 7$ patients $(28.7 \%)$. None of the patients needed reoperation. There was no postoperative mortality. The operative findings and perioperative outcomes are described in Table 2.

All the patients completed three cycles of PIPAC and histological response assessment was performed by an oncopathologist by the Peritoneal Regression Grading Score (PRGS). The four-tier PRGS is defined as Grade 1:

Table 1: Patients' characteristics and preoperative details

\begin{tabular}{lc}
\hline Variable & Value \\
\hline Number of patients & 7 \\
Sex (male/female) & $4: 3$ \\
Age, years (median) & 43 \\
Symptomatic: Asymptomatic & $3: 4$ \\
Primary & \\
Ovary & 2 \\
PPPC & 1 \\
Mesothelioma & 2 \\
Colorectal & 1 \\
Gastric & 1 \\
PCI (mean) & 17.4 \\
ECOG (median) & 1 \\
Previous surgery (\%) & $3(42.8)$ \\
Previous systemic chemotherapy & \\
$\geq 2$ lines & 2 \\
1 line & 5 \\
\hline PCI-Peipha conpos
\end{tabular}

$\mathrm{PCI}=$ Peripheral component interconnect, $\mathrm{ECOG}=$ Eastern Cooperative Oncology Group, PPPC=Primary papillary peritoneal cancer
Complete response; Grade 2: Major response; Grade 3: Minor response; and Grade 4: No response. ${ }^{[10]}$ One patient of there was complete histological remission; three patients had a partial response, one had stable disease and one patient had no response. In our study, the mean histological response curve showed that the response to is more pronounced after second PIPAC [Chart 1]. In one patient of mesothelioma, there was complete histological remission; three patients had partial response, one had stable disease, and one patient had no response with clinical progression [Figure 3].

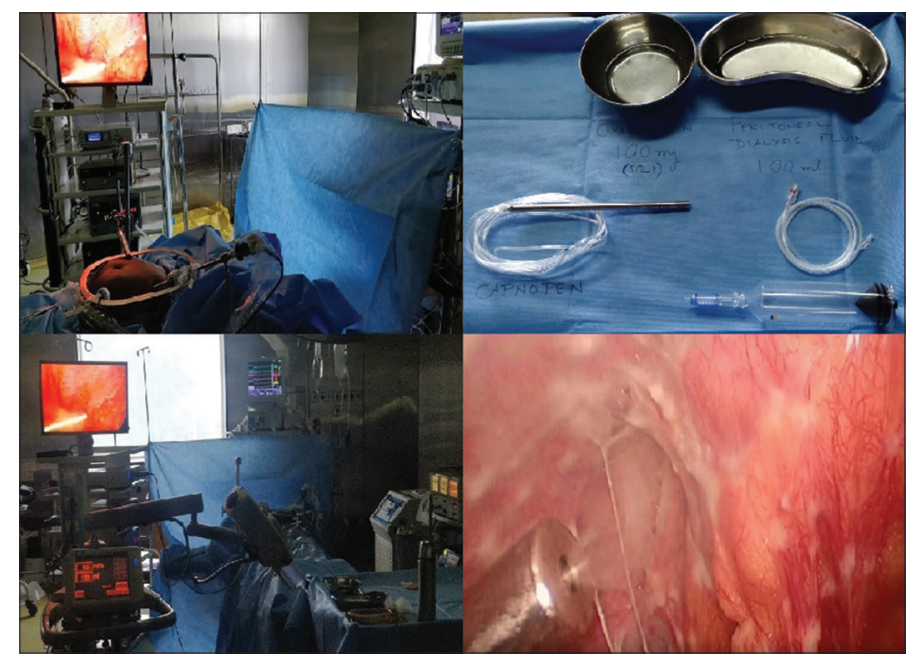

Figure 1: Operation theater setup during pressurized intraperitoneal aerosol chemotherapy procedure. All the operation theater personnel must be out during the procedure. The chemotherapy drug is sprayed intraperitoneally by the Capnopen which is connected to the high-pressure injector

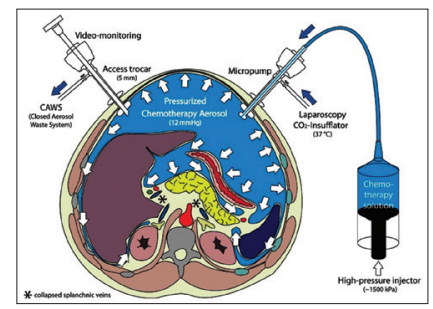

Figure 2: Diagrammatic representation of pressurized intraperitoneal aerosol chemotherapy (reproduced from reference 15 after permission Prof Marc Reymond)

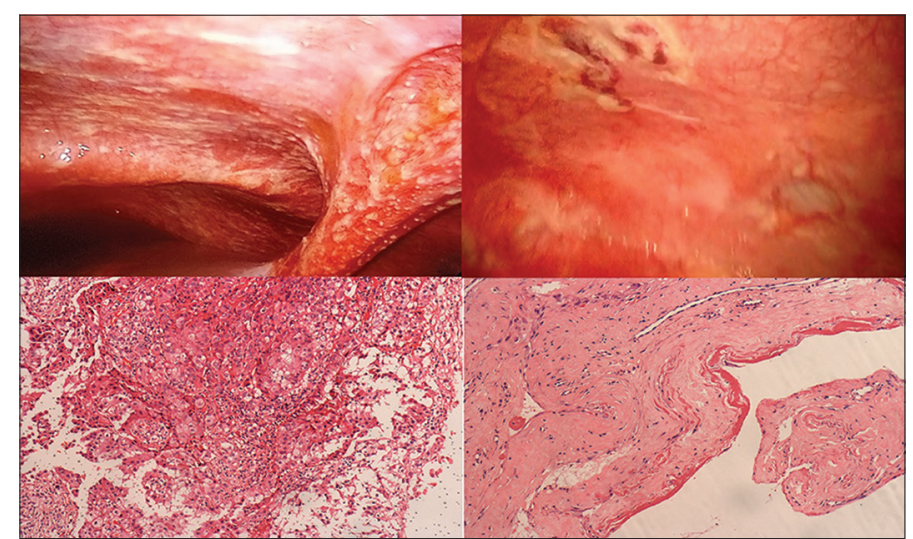

Figure 3: Macroscopic and histological response after pressurized intraperitoneal aerosol chemotherapy. A Fifty-two-year-old male patient with diffuse carcinomatosis from mesothelioma on laparoscopy and microscopy showed papillary fronds of mesothelial proliferation. After $3^{\text {rd }}$ pressurized intraperitoneal aerosol chemotherapy, there was disappearance of peritoneal nodules, and microscopy confirmed complete regression with the pathological complete response

South Asian Journal of Cancer • Volume 8 •Issue 1 • January-March 2019 
Table 2: Operative findings and perioperative outcomes

\begin{tabular}{|c|c|c|c|c|c|c|c|c|c|c|}
\hline Patient & Disease & $\begin{array}{l}\text { Previous } \\
\text { chemotherapy }\end{array}$ & $\begin{array}{c}\text { PCI } \\
\text { before } \\
\text { PIPAC }\end{array}$ & Ascites & $\begin{array}{c}\text { PIPAC } \\
\text { procedures }\end{array}$ & Chemotherapy used & $\begin{array}{c}\text { Operative } \\
\text { time } \\
(\mathrm{min})\end{array}$ & $\begin{array}{c}\text { Hospital } \\
\text { stay } \\
\text { (days) }\end{array}$ & $\begin{array}{l}\text { Adverse } \\
\text { effects } \\
\text { (CTCAE 1-4) }\end{array}$ & $\begin{array}{c}\text { Peritoneal } \\
\text { regression } \\
\text { grading } \\
\text { score }^{[10]} \\
\end{array}$ \\
\hline 1 & Colorectal & 2 lines & 17 & Absent & 3 & Oxaloplatin & 100 & 1 & Nil & 3 \\
\hline 2 & Ovary & 1 line & 23 & Present & 3 & Cisplatin + doxorubicin & 120 & 2 & Nil & 2 \\
\hline 3 & Mesothelioma & 1 line & 19 & Absent & 3 & Doxorubicin + cisplatin & 110 & 3 & Pain (2) & 1 \\
\hline 4 & Ovary & 2 lines & 16 & Absent & 3 & Cisplatin + doxorubicin & 80 & 3 & Vomiting (2) & 2 \\
\hline 5 & PPC & 1 line & 18 & Absent & 3 & Cisplatin + doxorubicin & 90 & 1 & Nil & 2 \\
\hline 6 & Gastric & 1 line & 11 & Absent & 3 & Doxorubicin + cisplatin & 100 & 2 & Pain (2) & 4 \\
\hline 7 & Mesothelioma & 1 line & 18 & Present & 3 & Doxorubicin + cisplatin & 90 & 1 & Nil & 2 \\
\hline
\end{tabular}

PIPAC=Pressurized intraperitoneal aerosol chemotherapy, PCI=Peripheral component interconnect, $C T C A E=C o m m o n$ terminology criteria for adverse events, PPC=Primary peritoneal cancer

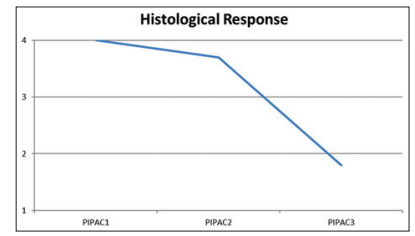

Chart 1: The four-tier Peritoneal Regression Grading Score was used to assess the response. ${ }^{[10]}$ In our study, the mean histological response curve showed that the response to is more pronounced after second pressurized intraperitoneal aerosol chemotherapy

\section{Discussion}

PIPAC is an innovative intraperitoneal chemotherapy concept that seems to enhance the effectiveness by taking advantage of the physical properties of gas and pressure. A "therapeutic capnoperitoneum" is created where aerosolized chemotherapy is sprayed in the carbon dioxide pneumoperitoneum. A minimum of three sittings is needed to observe any response. The aerosolization of the chemotherapy agent creates micron-size drug particles reducing the average diameter of a chemotherapeutic infusion and forms a thin film of microdroplets over the entire peritoneal cavity, increasing the contact surface area between drugs and tissues. PIPAC pharmacokinetics permits homogenous drug distribution with a minimal drug dose (1/10 the dose of systemic chemotherapy). The other advantages include easy to perform with minimal learning curve, good tolerance and multiple applications can be performed at 6 weekly intervals. It can be performed concurrently with systemic chemotherapy especially in patients with advanced stage of PC having ascites and complains of abdominal pain or subocclusive intestinal symptoms. In our series, local toxicity of PIPAC was acceptable even with repeated delivery. No patient developed bowel perforation, and no severe gastrointestinal symptoms were registered. In patients presenting with worsening quality of life because of peritoneal disease diffusion, the combination of the two treatments enables rapid symptom palliation with PIPAC. In our patients, symptomatic relief and ascites resolution were seen in all the patients.

The safety guidelines have been well established, and following this, set protocol ensures that PIPAC is safe, easily reproducible. Selection is important as patients with multiple abdominal surgeries, intestinal obstruction, and poor performance status are unlikely to tolerate or derive any benefit. PIPAC may not only be considered a palliative treatment, but in combination with systemic chemotherapy, with appropriate drug doses, it could possibly become part of the standard therapeutic course of PC.

South Asian Journal of Cancer $\bullet$ Volume 8 Issue 1 January-March 2019

\section{Conclusion}

Patients with advanced PC who are not candidates for curative resection have option of palliative systemic chemotherapy.

PIPAC is a simple, easily reproducible with no postoperative major toxicity, and the tolerance of the procedure is usually excellent. Although there is no Level 1 evidence as it can be used in the palliative setting, this combined treatment as well as being ethically accepted may be a useful strategy for these patients. Our results obtained in a small cohort of patients show safety and feasibility of PIPAC. Prospective studies are needed to expand the cohort of patients who can most benefit from this treatment. These studies may improve the technique and assess whether this combination therapy could become part of the standard treatment for PC.

\section{Declaration of patient consent}

The authors certify that they have obtained all appropriate patient consent forms. In the form the patients have given their consent for their images and other clinical information to be reported in the journal. The patients understand that their names and initials will not be published and due efforts will be made to conceal their identity, but anonymity cannot be guaranteed.

\section{Financial support and sponsorship}

Nil.

\section{Conflicts of interest}

There are no conflicts of interest.

\section{References}

1. Sugarbaker PH. Observations concerning cancer spread within the peritoneal cavity and concepts supporting an ordered pathophysiology. Cancer Treat Res 1996;82:79-100.

2. Sugarbaker PH, Ryan DP. Cytoreductive surgery plus hyperthermic perioperative chemotherapy to treat peritoneal metastases from colorectal cancer: Standard of care or an experimental approach? Lancet Oncol 2012;13:e362-9.

3. Beckert S, Struller F, Grischke EM, Glatzle J, Zieker D, Königsrainer A, et al. Surgical management of peritoneal surface malignancy with respect to tumour type, tumour stage and individual tumour biology. Zentralbl Chir 2016;141:415-20.

4. Jacquet P, Stuart OA, Chang D, Sugarbaker PH. Effects of intra-abdominal pressure on pharmacokinetics and tissue distribution of doxorubicin after intraperitoneal administration. Anticancer Drugs 1996;7:596-603.

5. Minchinton Al, Tannock IF. Drug penetration in solid tumours. Nat Rev Cancer 2006;6:583-92.

6. Tempfer CB, Winnekendonk G, Solass W, Horvat R, Giger-Pabst U, Zieren J, et al. Pressurized intraperitoneal aerosol chemotherapy in women with recurrent ovarian cancer: A phase 2 study. Gynecol Oncol 2015; 137:223-8.

7. Nadiradze G, Giger-Pabst U, Zieren J, Strumberg D, Solass W, Reymond MA, et al. Pressurized intraperitoneal aerosol chemotherapy (PIPAC) with 
low-dose cisplatin and doxorubicin in gastric peritoneal metastasis. J Gastrointest Surg 2016;20:367-73.

8. Demtröder C, Solass W, Zieren J, Strumberg D, Giger-Pabst U, Reymond MA, et al. Pressurized intraperitoneal aerosol chemotherapy with oxaliplatin in colorectal peritoneal metastasis. Colorectal Dis 2016;18:364-71.

9. Solass W, Giger-Pabst U, Zieren J, Reymond MA. Pressurized intraperitoneal aerosol chemotherapy (PIPAC): Occupational health and safety aspects. Ann Surg Oncol 2013;20:3504-11.

10. Solass W, Sempoux C, Carr NJ, Detlefsen S, Bibeau F. Peritoneal sampling and histological assessment of therapeutic response in peritoneal metastasis: Proposal of the peritoneal regression grading score (PRGS). Pleura Peritoneum 2016;1:99-107. 